\title{
Reflets
}

Revue d'intervention sociale et communautaire

\section{Militer pour l'accès des personnes à mobilité réduite dans les Maritimes}

\section{Entrevue avec Daniel Savoie}

\section{Isabelle Côté}

Volume 23, numéro 2, automne 2017

URI : https://id.erudit.org/iderudit/1043301ar

DOI : https://doi.org/10.7202/1043301ar

Aller au sommaire du numéro

Éditeur(s)

Reflets, Revue d'intervention sociale et communautaire

ISSN

1203-4576 (imprimé)

1712-8498 (numérique)

Découvrir la revue

Citer ce document

Côté, I. (2017). Militer pour l'accès des personnes à mobilité réduite dans les Maritimes : entrevue avec Daniel Savoie. Reflets, 23(2), 16-30.

https://doi.org/10.7202/1043301ar d'utilisation que vous pouvez consulter en ligne.

https://apropos.erudit.org/fr/usagers/politique-dutilisation/ 


\section{Militer pour l'accès des personnes à mobilité réduite dans les Maritimes}

\section{Entrevue avec Daniel Savoie, militant acadien, Professeur retraité, Université du Nouveau-Brunswick}

Cette entrevue a été réalisée le 19 août 2017 par Isabelle Côté et rédigée par Isabelle Côté.

\section{Biographie}

Daniel Savoie est professeur retraité de l'Université du Nouveau-Brunswick, où il a enseigné pendant 18 ans à la Faculté des sciences infirmières. Fièrement acadien, il milite depuis plusieurs années afin d'accroître l'accessibilité des personnes à mobilité réduite dans différents milieux. Il a d'ailleurs obtenu, en 2008, le CNA Nurse to Know : Centennial Achievement Award \& CNA Centennial Award, décernés pour son leadership et sa contribution à l'avancement des soins infirmiers au pays. M. Savoie demeure présentement à l'Île-du-Prince-Édouard avec sa conjointe.

Reflets: Bonjour, Monsieur Savoie. Nous vous remercions d'accepter de contribuer à ce numéro de Reflets portant sur le militantisme et le changement social. Dans un premier temps, pourriez-vous nous décrire votre parcours comme personne militante? En d'autres termes, qu'est-ce qui vous a amené à militer?

D. Savoie : Tout a débuté en 1988 lorsque j'ai eu un accident de voiture qui m’a laissé paraplégique. J'ai immédiatement découvert toutes les barrières et tous les défis qui s'imposent aux personnes à mobilité réduite, car j'étais moi-même confronté à ces difficultés. À l'époque, je venais tout juste de terminer ma deuxième année universitaire en soins infirmiers et automatiquement la question a été : est-ce qu'il est capable de terminer son baccalauréat en fauteuil roulant au département des sciences infirmières de 
l'Université de Moncton? C'était du jamais vu pour eux; ils n'avaient jamais vu un infirmier en fauteuil roulant à l'hôpital auparavant. Habituellement, les gens changent de domaine ou de carrière. Pour une raison ou pour une autre, mes professeurs à l'Université et la doyenne de l'École des sciences infirmières ont approchél'Association des infirmières et infirmiers du NouveauBrunswick. Ils auraient eu une longue discussion et ils m'ont finalement permis de continuer mes études.

J'ai quand même été chanceux parce que j'étais anciennement militaire. Donc, j'étudiais par l'entremise du Programme de formation des officiers de la Force régulière des Forces canadiennes, ce qui me permettait de payer ma scolarité, les livres, etc. Suite à mon accident, j'ai obtenu une assurance invalidité prolongée, et cette assurance m'a permis de payer mes cours. Étant donné que j'étais en fauteuil roulant, j'avais besoin de one-on-one avec des professeurs, d'où l'importance du support. J'avais non seulement le support de mes professeurs pour pouvoir poursuivre mes études ainsi que le désir de lever les barrières et d'affronter les défis, mais j'avais également le support financier qui me permettait de me payer un professeur one-on-one.

Donc, j'ai poursuivi mon baccalauréat en fauteuil roulant. J'ai fait tous mes cours de troisième année, mais ces cours étaient surtout axés sur la santé mentale, l'obstétrique, etc. En quatrième année, quand est venu le temps d'entamer mes stages en chirurgie médicale, c'était le fun, car ils ont adapté mon programme. J'ai donc eu l'opportunité d'aller travailler en réadaptation et puis, pour moi, ça a été le début de mon militantisme.

Reflets : En quoi le début de votre carrière est-il en lien avec l'émergence de votre militantisme?

D. Savoie : Le militantisme a débuté lorsque j'ai eu l'opportunité de travailler dans un département de réadaptation où les gens avaient besoin d'aide. Au fil des années, j'ai rencontré énormément de personnes. Certaines d'entre elles étaient davantage fortunées et, comme moi, avaient eu accès soit à l'éducation, soit à des 
ressources financières, soit à une forme ou une autre de soutien social. Par contre, plusieurs autres n'avaient accès à rien ou avaient accès à peu de ressources.

Donc, ce sont ces gens-là qui avaient le plus besoin d'aide, car ils avaient des difficultés à vocaliser ou à nommer leurs besoins ou encore à trouver des moyens pour franchir les barrières qui se dressaient devant eux. Certains d'entre eux sont nés avec un handicap et ont toujours été couvés par une mère poule qui s'occupait toujours d'eux et, donc, ils n'avaient jamais vraiment appris à se débrouiller par eux-mêmes. D'autres ont vécu le handicap à partir de l'adolescence ou de l'âge adulte et n'ont jamais eu un parent, un ami ou un professionnel qui s'est vraiment occupé d'eux ou qui leur a montré ou indiqué comment se débrouiller par eux-mêmes.

Vous savez, il y a des choses que l'on apprend dès notre jeune âge. Quand un défi ou une difficulté nous tombe sur la tête et qu'on trouve ça lourd, soit qu'on affronte le problème ou qu'on évite le problème : fight or flight. Ceux qui affrontent le problème vont utiliser tous les moyens appris au cours de leur vie afin de gérer ou de s'adapter à la situation. En tant que professionnel de la santé avec un handicap, j'ai découvert que plusieurs personnes n'ont pas ces moyens et ne savent pas comment affronter leurs problèmes. C'est pour ça que j'ai passé beaucoup de temps à faire du bénévolat, à éduquer les gens, à tenter de les aider, à essayer de comprendre leurs besoins, à trouver des ressources et à toujours aller au-delà, afin que les individus avec un handicap soient en mesure d'affronter ou de relever les défis qui s'imposent à eux.

Donc, oui, j'ai fait énormément de bénévolat pour aider ces gens, mais il y avait tout le côté de ma situation personnelle. Parce que, moi, j'avais vingt ans au moment de mon accident : tout était nouveau pour moi. Il a fallu que j'apprenne bien des choses. Par contre, j'ai découvert qu'il y a de nombreux défis que l'on peut surmonter, mais surtout que de les surmonter 
pouvait non seulement m'aider, mais aider d'autres personnes aussi. C'est donc à ce moment-là que j'ai commencé à militer plus activement.

Reflets: Votre militantisme part donc d'une situation personnelle, et on comprend assez bien pourquoi vous avez fait le choix de militer plus spécifiquement pour favoriser l'accès des personnes à mobilité réduite. Est-ce que vous pourriez nous décrire vos batailles les plus significatives au fil des années, ainsi que des gains obtenus?

D. Savoie : Je dirais que mes revendications ont touché deux aspects de ma vie : ma carrière et ma vie personnelle.

Du point de vue de ma carrière professionnelle, j'ai remarqué qu'aucune association ou aucun organisme ne se penchait sur le développement de la profession des soins infirmiers dans le domaine de la réadaptation au Canada. C'était difficile parce qu'on parle de tenter d'ouvrir de nombreuses portes qui n'ont jamais été ouvertes par le passé. J'ai donc participé au développement d'un programme de certification pour les infirmières et infirmiers travaillant dans le domaine de la réadaptation. Il s'agissait spécifiquement d'un programme de certification sous l'Association des infirmières et infirmiers $\mathrm{du}$ Canada, permettant aux professionnelles et professionnels de se certifier après un certain nombre d'heures. Toujours en ce qui a trait à ma carrière, j'ai aussi été le fondateur et le premier président de l'Association des infirmiers et infirmières en réadaptation. Donc, tout un travail dans le domaine de l'éducation des gens dans la même profession que moi. En d'autres mots, il y a eu énormément de travail qui s'est fait dans le domaine de l'éducation des gens qui ont la même profession que moi.

En ce qui a trait à ma vie personnelle, mon militantisme s'est surtout déployé au niveau de l'accessibilité des gens à mobilité réduite. Par exemple, au Nouveau-Brunswick, il n'y avait aucun endroit où les personnes avec un handicap pouvaient jouer au golf : ça n'existait pas. Donc un jour, je me suis dit que ça serait 
agréable d'aller jouer au golf avec mes amis. J'ai donc commencé à m'informer des options qui s'offraient à moi, puis le constat a été que d'aller jouer au golf, ce n'était pas possible. Étant qui je suis, ayant les ressources pour apprendre comment je devais m'y prendre, j’ai commencé à étudier les besoins, mais aussi à examiner ce qui existait déjà en termes de ressources. J'ai découvert qu'il y avait au Canada un véhicule adapté qui permet à une personne comme moi, en fauteuil roulant, d'embarquer sur le véhicule et d'aller jouer au golf comme toute autre personne : ce véhicule permet de me lever debout, de frapper la balle en position debout, d'aller sur les verts, etc. Or, je ne savais pas comment aller chercher les fonds. Donc, qu'est-ce que j'ai fait? J'ai approché différents organismes et je me suis associé avec une personne qui savait comment aller chercher les fonds. Cette personne-là a ciblé de nombreuses compagnies qui pourraient potentiellement être intéressées à investir dans le projet. On a donc approché un concessionnaire en lui demandant s'il était prêt à commanditer une partie du véhicule, et il a finalement offert de le payer en totalité. Après quelques mois, ce véhicule était disponible sur le terrain de golf, ce qui permettait aux gens dans une situation similaire à la mienne de dorénavant aller jouer au golf.

Cette stratégie, je l'ai employée par la suite pour mes autres batailles. Parce que quand tu milites, il faut : 1) que tu identifies le problème; 2) que tu identifies ce que tu dois faire pour le résoudre; 3) que tu ailles chercher les ressources qui vont te permettre de passer à l'action; 4) que tu réévalues régulièrement tes approches.

Je vais vous donner un autre exemple, toujours en ce qui a trait à l'accessibilité dans les sports. Je suis un ancien militaire et j'ai toujours voulu tirer à la carabine dans un champ de tir. À l'Île-du-Prince-Édouard, ce n'est pas possible : tous les champs de tir ont été façonnés pour les gens qui n’ont aucun handicap. Qu'est-ce que j'ai fait? J'ai identifié le besoin de localiser un champ de tir accessible. Pour ça, je suis allé rencontrer des gens à ParaSport and Recreation PEI. Je leur ai fait part de mon projet 
d'avoir un champ de tir adapté à l'Île-du-Prince-Édouard, de même que j'ai vraiment essayé de leur vendre l'idée du besoin d'avoir un champ de tir adapté à l'Île-du-Prince-Édouard. Après ça, on a trouvé les fonds, on a acheté les carabines et on a été en mesure de développer le programme avec des carabines en provenance de l'Allemagne : les mieux cotées du point de vue du sport. Elles ne sont pas adaptées; c'est la position, la table, le spring qui le sont. Comme les gens avec un handicap ont différents besoins, on est en mesure d'adapter leur table de tir en fonction de leur handicap. Par exemple, un de mes amis n'a pas la force de soulever sa carabine. Il peut alors utiliser un spring attaché à une table qui lui permet de soutenir sa carabine. Grâce à cela, il peut maintenant pratiquer son sport.

Enfin, une de mes batailles significatives - et la plus récente — a été l'installation de rampes d'accès sur la plage Stanhope à l'Île-du-Prince-Édouard. Les gens à mobilité réduite ont de nombreuses difficultés à circuler sur les plages. Par exemple, en fauteuil roulant, une fois que tu arrives sur le sable, tu es mal pris. Tu ne peux aller nulle part. C'est plate parce que tout le monde s'en va à la plage, mais, nous, on doit rester sur la falaise. Donc, pour notre projet, nous avons identifié le besoin de rendre les plages accessibles aux personnes à mobilité réduite à l'̂̂le-du-Prince-Édouard. Il fallait identifier comment on pouvait solutionner le problème. Tout a débuté quand mon épouse et moi étions allés dans les Caraïbes il y a environ trois ans et avions découvert qu'il y avait des fauteuils adaptés de disponibles sur leurs plages. Nous avons aussi identifié un genre de tapis construit par les militaires américains, qui permet à certains de leurs véhicules d'aller sur les plages. Ce produit est maintenant utilisé un peu partout dans le monde afin de permettre aux personnes à mobilité réduite de se déplacer sur les plages. Étant donné que c'est difficile de négocier en son nom personnel, j’ai dû identifier un organisme avec lequel je me suis associé, un organisme très connu à l'Île-du-Prince-Édouard qui soutient les gens avec un handicap : le Spinal Cord Injury — Prince Edward 
Island. J'ai approché les responsables de cette association pour leur demander s'ils seraient intéressés à s'associer à moi pour sensibiliser Parcs Canada à l'importance de rendre les plages accessibles aux gens à mobilité réduite. Ensemble, nous avons approché des représentants de Parcs Canada : nous leur avons présenté le projet, les besoins spécifiques des gens à mobilité réduite, de même que les différentes options qui permettent aux touristes ayant un handicap de s'amuser sur les plages à l'île-duPrince-Édouard et de bénéficier de celles-ci. De même, nous avons mis l'accent sur l'accessibilité des plages à l'ensemble de la population, ainsi qu'aux nombreux touristes qui s'y rendent chaque année. Ce projet bénéficie non seulement aux gens ayant un handicap, mais aussi à tous ceux et celles qui peuvent difficilement accéder aux plages. Par exemple, une famille avec une poussette. On a donc soulevé l'importance de l'accessibilité, et ça n'a pas pris de temps que les fonds ont été trouvés et, depuis cette année, la plage de Stanhope est complètement accessible. Nous avons l'intention de travailler avec Parcs Canada pour rendre plusieurs autres plages accessibles à l'ensemble de la population d'ici les prochaines années.

Reflets : Ça a d'ailleurs été médiatisé, non?

D. Savoie: Il y a eu plusieurs reportages par rapport à ça. Ça a passé à Radio-Canada; j'ai été interviewé à la radio entre autres. Cette bataille, j'en bénéficie, mais ce n'était pas nécessairement juste pour moi : je constate que ça aide énormément de gens dans la même situation ou dans une situation pire que la mienne. La seule différence, c'est que je suis capable de trouver les gens qui peuvent m'aider, les ressources nécessaires, etc.

Reflets: Dansce contexte-là, vous avez certains privilèges qui vous permettent de militer et d'obtenir des gains?

D. Savoie : Je ne sais pas si on peut parler de privilèges. Peut-être que oui? Je dirais que je possède certaines aptitudes que j'ai apprises tout au long de ma vie. Mes parents, mon père comme ma mère, ont toujours été des gens très forts de caractère. Ils m’ont appris 
que, lorsqu'on fait face à des obstacles au cours de notre vie, il existe toujours des moyens de moyenner: When there's a will, there's a way. Il faut juste trouver la façon de faire. Tant et aussi longtemps que tu as la volonté, tu es capable de te mettre en action. Je vous donne un exemple. Ma conjointe et moi aimons beaucoup voyager. Dans le cadre de nos voyages, nous avons découvert qu'une panoplie d'obstacles se dressait devant nous du fait que je suis en fauteuil roulant. Si on s'était toujours penché sur le côté négatif, on aurait simplement arrêté de voyager, vous voyez? On s'est plutôt dit : "Wow, il y a beaucoup de choses à faire dans le monde. Penchons-nous sur ce que nous pouvons faire, sur ce que nous sommes capables de faire. "C'est là qu'on a investi nos énergies.

J'ajouterais à ça que ce qui m'a permis d'ouvrir des portes et ça a très bien fonctionné pour moi —, c'est mon handicap. J'ai pu m'éduquer et j'ai réalisé qu'en fauteuil roulant, j'ai parfois l'opportunité de me faire remarquer. Les gens me prêtent attention et donc prêtent attention à ma cause parce que $\mathrm{j}$ ' attire automatiquement l'attention, ce qui me permet de vendre ma salade. Puis ça, je dirais que ça m'aide beaucoup.

Reflets: Outre le fauteuil roulant, est-ce que d'autres facteurs expliquent pourquoi vous avez été en mesure d'avoir cette crédibilité et d'obtenir autant de gains suite à vos batailles?

D. Savoie : Oui. Avec le fauteuil roulant, j'attire l'attention des gens. Mais, l'autre élément qui m'a permis de militer, ce sont les aptitudes que j'ai acquises tout au long de ma vie pour résoudre un problème ou une situation, par exemple, comme je l'ai dit auparavant, trouver le soutien nécessaire, identifier les ressources pour m'appuyer et trouver les outils pour relever les défis. Donc, ce côté-là de ma personnalité. Mon éducation a aussi aidé, tant les ressources pratiques que théoriques. Je dirais que je suis dans une situation avec deux côtés d'une médaille : j'ai différents besoins d'un point de vue personnel en tant que patient ou client, mais j'ai aussi le côté professionnel qui a énormément aidé. 
Reflets : Donc, le statut par exemple peut aider?

D. Savoie : Oui, le statut professionnel. Quand j'ai approché les représentants de Parcs Canada avec le Spinal Cord Injury - Prince Edward Island, j'avais acquis une crédibilité que je n'aurais pas eue si je leur avais simplement dit : "Heille, je suis Monsieur X en fauteuil roulant et j'aimerais vous proposer un projet. " La crédibilité et le statut sont des éléments importants quand on milite. J'ai rencontré de nombreuses personnes en fauteuil roulant qui veulent militer comme moi, qui ont identifié un problème particulier, mais qui ne sont pas capables de faire le reste. Il faut être en mesure de mettre en place certaines stratégies et d'apprendre à utiliser les outils nécessaires pour se mettre en action et pour pouvoir faire une différence.

Reflets : D'où vous vient ce désir de vous investir autant dans une cause?

D. Savoie: Je crois fortement que tout le monde qui milite a une raison pour le faire. Et je crois sincèrement que c'est relié à un besoin, à certaines valeurs qu'on apprend tout au long de notre vie. Je crois que le fait qu'on désire militer, c'est relié soit à un besoin personnel ou à quelque chose qu'on aime faire. Vous savez, les gens qui vont dans les pays du tiers-monde pour aider des personnes moins fortunées ou qui s'impliquent dans les soupes populaires, qui veulent aider, selon moi, ils ont toujours une raison pour le faire. Il y a un lien quelque part, un lien avec leur vie personnelle, avec un ami, avec un parent ou avec une situation qui les pousse à se mettre en action. Peut-être aussi que c'est parce qu'ils ont appris l'importance d'aider quand ils étaient jeunes.

Dans mon cas, $j$ 'ai appris ça de mes parents. Je me souviens quand j'étais jeune - j'avais à peu près neuf ans — il y avait une famille vraiment pauvre par chez nous, dans notre village. Ils avaient vraiment été laissés à part pour Noël. Bien, je me souviens qu'après la messe de minuit, cette famille marchait toute seule sur le bord de la rue. Mes parents se sont arrêtés et ont 
embarqué tout le monde : on était à peu près huit ou neuf dans la voiture. Une chance que papa avait une Lincoln Continental : un méchant bateau! Mes parents les ont amenés chez nous pour qu'ils passent le réveillon de Noël avec nous. On a partagé notre dinde avec cette famille ainsi que nos cadeaux avec les enfants. C'était gros pour moi à cet âge-là, mais, en même temps, nos parents nous ont démontré qu'il fallait partager et qu'il fallait aider autrui et que si on est capable de le faire, il faut le faire.

Puis je pense que c'est un peu pour ça qu'encore aujourd'hui, quand je vois qu'il y a un besoin, je me mets en action. Le fait, par exemple, que les gens puissent utiliser le tapis sur les plages, ça répond à un besoin intérieur qui m’apporte tellement de satisfaction. Justement l'autre jour, j'ai vu un monsieur qui était assis sur un fauteuil roulant de plage sous un parasol. Je ne me suis pas dit : "Wow, j'ai accompli cela! " J'ai plutôt ressenti : "Wow, cet homme est maintenant capable d'aller sur la plage! » J'étais tellement fier qu'il puisse en bénéficier. Encore une fois, pas fier que ce soit moi qui l'aie développé, mais, plutôt, content pour lui. Je pense que ce genre de situation vient me chercher depuis le Noël où mes parents ont partagé le réveillon avec cette famille; ils m'ont appris à faire des choses pour ceux qui en ont besoin. J'ai toujours aidé, j'ai toujours tenté de faire une différence à plusieurs niveaux.

Reflets: Dans cette quête de changement, pourriez-vous identifier les principaux enjeux ou défis que vous avez rencontrés dans votre parcours de militant?

D. Savoie : Le plus grand défi, je vous dirais, c'est le manque de sensibilité de certaines personnes. Il y a des gens qui ne sont pas sensibilisés aux défis que les personnes à mobilité réduite peuvent rencontrer dans leur quotidien. Par exemple, j'ai milité dans le passé afin de rendre notre communauté plus accessible aux personnes avec un handicap. Certaines personnes qu'on approchait dans les restaurants ou les magasins démontraient une grande ouverture 
parce que, dans le passé, elles ont connu quelqu'un, soit un parent ou un ami, qui avait un handicap ou une limitation. Par contre, il y avait d'autres gens qui étaient complètement ignorants. Dans le sens que c'était la première fois qu'ils voyaient une personne en fauteuil roulant. Donc ça, c'est un des grands défis. Il fallait éduquer et sensibiliser les gens, mais aussi les amener à comprendre les besoins spécifiques de cette population.

Parfois, il suffit d'une personne sensibilisée à l'intérieur d'un groupe pour faire la différence. Je crois que c'est peut-être une des raisons pour lesquelles ça a si bien été avec Parcs Canada. Il existe cependant d'autres personnes qui sont plutôt en réaction : "Quoi? On doit dépenser 15000 \$ pour quoi encore? » Vous savez, ce genre d'attitude? Et c'est là que réside un des principaux défis. Parce qu'à moins que cette personne-là se retrouve en fauteuil roulant et devienne sensibilisée à la cause, c'est un travail qu'on doit faire nous-mêmes. Donc, la sensibilisation, c'est un grand défi. Malgré tout, même avec ce type de personnes, si tu leur parles de ta cause et que tu les amènes à voir le besoin, ça peut marcher dépendamment de ton approche. On doit simplement être préparé avant d'en parler.

Reflets : Pouvez-vous nous expliquer ce que vous entendez par cela?

D. Savoie : En fait, j'ai appris dans la vie qu'on ne peut pas expliquer notre cause " à peu près " parce que nos résultats vont aussi être juste des « à peu près ". Il faut vraiment que tu sois en mesure de leur démontrer pourquoi c'est important pour eux de s'occuper du problème et pourquoi ils doivent le faire maintenant. Puis ça, je pense que c'est mon côté académique qui m’a amené à développer des stratégies pour être en mesure de le faire. Vous savez, quand on rédige des demandes pour aller chercher des octrois financiers, il faut être capable de justifier en quoi le projet est pertinent. C'est la même chose quand on mène des batailles. Donc, je mets en œuvre toutes les habiletés que j’ai développées en tant que professionnel quand je milite. 
Reflets : Est-ce quilly a d'autres enjeux ou défis auxquels vous êtes confronté?

D. Savoie : C’est certain que le côté monétaire, c'est toujours un défi. Dans ce contexte, j'ai appris qu'il faut toujours se munir d'un plan B quand on va négocier. Le plan $B$, ça fonctionne vraiment bien. Par exemple, quand j'ai approché les gestionnaires du terrain de golf pour qu'ils puissent adapter leur terrain, je savais qu'il s'agissait d'une compagnie à but lucratif. Je devais justifier combien de clients ça pourrait leur apporter et pourquoi ça valait la peine pour eux de rendre leur terrain accessible. Ils étaient un peu hésitants, donc je leur ai parlé de mon plan B : «Écoutez, je comprends l'aspect financier, mais $\mathrm{j}$ 'ai trouvé du financement pour le véhicule en question. » Là, tout d'un coup : " Ah? Ça pourrait nous aider ça, on pourrait faire de la promotion..." Là, tout d'un coup, ça change la donne.

Reflets: C'est une excellente stratégie! Donc, vous leur montrez ce que ça peut leur apporter à eux aussi?

D. Savoie: Ah oui, en effet! Je dois toujours leur présenter le pourquoi du besoin. C'est pas seulement ce que ça peut apporter aux personnes ayant un handicap, mais aussi comment ils peuvent en bénéficier. Quand on milite, c'est ça qu'il faut faire : être en mesure de présenter pourquoi le projet va générer des bénéfices pour eux. Quand on se met en action, quand on développe un projet, quand on va chercher de l'argent, ce n'est pas nos besoins qu'on doit mettre de l'avant : ce sont leurs besoins. C'est vraiment ça qu'il faut faire : il faut parler leur langage, aller chercher la terminologie qui va les accrocher, vérifier leurs objectifs, regarder comment on devrait leur présenter le projet, analyser ce qui est important pour eux. Quand tu arrives pour les rencontrer et que tu présentes ta cause et les besoins que tu as identifiés, il faut que tu sois en mesure de leur faire comprendre en quoi ta cause, c'est leur cause finalement. C'est vraiment ça.

Puis, des fois, il faut présenter notre projet comme un projet qui va leur apporter plus à eux qu'aux personnes pour qui on milite. Il faut que tu leur présentes ainsi : «Voici ce dont vous 
avez besoin et voici pourquoi vous avez besoin de moi pour vous aider. "Ça a été ça mon astuce pour le terrain de golf. Je leur ai apporté le fait qu'ils seraient le premier terrain de golf accessible en Atlantique, que leur terrain apparaîtrait sur tous les dépliants de Golf Canada, etc. Tout d'un coup, ils voyaient toute la question du statut.

Reflets : Cela fait qu'eux voient le prestige, mais aussi l'argent qui pourrait rentrer?

D. Savoie: Bien absolument! Ils voient les deux : le prestige et l'argent. Ça a été la même chose pour Parcs Canada. Donc, pour qu'ils puissent voir le prestige et l'argent, il fallait aussi démontrer que le projet allait bénéficier à un grand nombre de personnes : tu ne vas pas seulement parler en ton nom personnel ou au nom de ton groupe. Pour la question de l'accessibilité des plages, il y a un bon pourcentage de gens à mobilité réduite à l'îledu-Prince-Édouard, mais on a présenté le projet de manière à englober toutes les catégories de personnes qui pourraient en bénéficier : les personnes âgées qui ont un problème de mobilité, les familles avec de jeunes enfants en poussettes, etc. Donc, on identifie tous les groupes qui vont bénéficier du projet et on tente d'aller chercher des appuis d'organismes à travers ça. On essaie de mettre plus de poids dans notre balance.

Reflets : Vous laissez entendre que votre militantisme part de certains enjeux que vous vivez sur une base individuelle et que vous tentez ensuite d'apporter ces enjeux à des gens qui ont un pouvoir d'influence en leur démontrant comment votre problème en affecte d'autres. Quels liens faites-vous entre votre militantisme et le changement social?

D. Savoie : Quand on parle de changement social, tout ce que je fais est en lien avec le changement social. Si on regarde vingt, quarante ou cinquante ans passés, quelqu'un qui avait un handicap restait à la maison. Si on remonte un peu plus loin en arrière, on les cachait. Et ça, ça a changé. Du point de vue du changement social, la situation des personnes à mobilité réduite a changé depuis de nombreuses années, et ça change encore. On continue 
de sensibiliser la population à l'importance de continuer dans cette voie. On voit plusieurs personnes en fauteuil roulant un peu partout maintenant, mais, si on regarde dans d'autres pays, ce n'est pas nécessairement le cas. Au Canada, ça a changé, mais, encore là, ça dépend où tu habites et quelles personnes t'entourent. Mais lorsqu'on milite pour la cause et qu'on est en contact avec les personnes qui prennent les décisions, je pense que ce qu'on fait est directement lié au changement social.

Reflets: Comme on parle du changement social, est-ce que, selon vous, les travailleuses et travailleurs sociaux ont un rôle à jouer dans la cause de l'accessibilité et, si oui, qu'est-ce que pourrait être ce rôle?

D. Savoie: Vous savez, quand on parle de cette cause, je ne pense pas que ça fait une différence si c'est le travailleur social, l'infirmière, le médecin ou le psychologue : je pense qu'on a tous un rôle à jouer dans l'aide apportée ou le support face à une cause. Si on regarde spécifiquement les travailleuses et travailleurs sociaux, vous travaillez énormément au niveau du changement social et vous avez un rôle important par rapport à ça, mais je ne pourrais pas vous dire quoi faire ni comment le faire. Je crois, par contre, qu'on devrait parler davantage de comment on s'associe à une cause, quel est le processus pour améliorer cette cause et comment faire. Et je pense aussi qu'il y aurait des avantages à ce que l'on enseigne ceci dans différentes disciplines, que ce soit en soins infirmiers, en médecine... Il est très important de s'associer à l'approche multidisciplinaire, même quand on milite, pour bien comprendre la cause. C'est un côté que tout baccalauréat devrait avoir : comment contribuer au changement social. Éventuellement, on doit pouvoir montrer à nos clients comment ils peuvent s'y prendre s'ils souhaitent eux-mêmes s'associer à une cause.

Reflets : En guise de conclusion, vous avez dit tantôt que votre militantisme vous apporte beaucoup sur le plan personnel, que ça vous fait chand au coeur de constater que les changements auxquels vous contribuez font une différence pour vous, mais aussi dans la vie des gens. Donc, le 
militantisme vous apporte aussi quelque chose sur les plans familial, professionnel et social, par exemple?

D. Savoie : Je pense qu'il y a un parallèle entre toutes ces composantes. Je crois que c'est en lien avec les deux côtés de la médaille dont je vous parlais tantôt. Je ne suis pas seulement une personne qui a découvert c'est quoi de vivre en fauteuil roulant. Oui, je vis les défis, les besoins, tout ce qui est de personnel, mais les autres aspects vont ensemble. De voir quelqu'un satisfait, de voir une personne qui bénéficie d'une mesure d'accessibilité, qui est heureuse, moi ça m'apporte une grande satisfaction personnelle.

Reflets : Ça a été très intéressant d'échanger avec vous; nous vous remercions pour votre temps!

Pour consulter les entrevues accordées par Daniel Savoie dans le cadre de l'installation des rampes d'accès sur les plages de l'Île-du-Prince-Édouard, vous pouvez cliquer sur les liens suivants :

\section{Entrevues accordées à la CBC le 13 juillet 2017 :}

- http://www.cbc.ca/player/play/995643459544

- http://www.cbc.ca/news/canada/prince-edward-island/pei-beachwheelchair-1.4201886

\section{Entrevue accordée à Radio-Canada le 31 juillet 2017 :}

- http://ici.radio-canada.ca/premiere/emissions/le-reveil-ile-du-prince-edouard/ segments/entrevue /32997/reveilipe-plages-accessibles 